\title{
Unreviewed Reports
}

\section{Intraperitoneal rupture of the bladder and ECT}

A 74 year old man with recurrent unipolar depression developed abdominal pain after the tenth electroconvulsion treatment. At laparotomy 31 of clear fluid was removed from the peritoneal cavity and a $6 \mathrm{~cm}$ bladder laceration sutured; recovery was uneventful. Three years previously, after negative results of investigations for retention, micturition had returned to normal; two months previously he had had urinary incontinence. He had received no tricyclic antidepressants for over a year. Though the association between ECT and ruptured bladder is rare, particular care should be taken in giving ECT to patients with prostatism, urinary difficulty, or retention.-A D IRVING, A M DRAYSON, Stracathro Hospital, Brechin, Angus DD9 7QA. (Accepted 30 November 1983)

\section{Sulphasalazine induced oral lichen planus}

A 51 year old man with rheumatoid disease started taking sulphasalazine $2 \mathrm{gm}$ daily while concurrently taking indomethacin. Four months later buccal ulceration developed. Biopsy confirmed lichen planus. A prednisolone mouthwash and stopping indomethacin did not help. Sulphasalazine was then stopped and the lesions resolved. Rechallenge with sulphasalazine produced ulceration within five days; it was stopped, and the lesions resolved. Non-steroid anti-inflammatory drugs, gold, chloroquine, and dapsone, all treatments used in rheumatoid arthritis, have been reported to cause oral lichen planus, but not sulphasalazine. Lichen planus in ulcerative colitis has been described, though sulphasalazine was not thought to be the causative factor. ${ }^{-}-\mathrm{P}$ T DAWES, $M$ F SHADFORTH, Staffordshire Rheumatology Centre, Haywood Hospital, Burslem, Stoke on Trent, Staffordshire. (Accepted 7 December 1983)

${ }^{1}$ Wyatt EH. Lichen planus and ulcerative colitis. Br F Dermatol 1975;93:465-8.

\section{Water intoxication in cranial diabetes insipidus treated with desmopressin}

A 26 year old woman treated for 15 years with pitressin tannate and three years with desmopressin (10-20 $\mu \mathrm{g}$ intranasally daily) was admitted unconscious with a 10 day history of headache and vomiting. She had a low serum sodium concentration $(111 \mathrm{mmol}(\mathrm{mEq}) / \mathrm{l})$ and osmolality $(231 \mathrm{mmol}(\mathrm{mosmol}) / \mathrm{kg})$ and high urine osmolality $(346 \mathrm{mmol} / \mathrm{kg})$. Water intoxication was diagnosed, she was treated with fluid deprivation, and after a diuresis regained consciousness, with normal serum sodium and osmolality. Though water intoxication has not been reported with intranasal desmopressin, and though ingestion of a large fluid load or deliberate overdosage cannot be excluded, the former may have been responsible.-J ROWE, Sandwell District General Hospital, West Bromwich B71 4JH. (Accepted 7 December 1983)

\section{Resolution of membranous glomerulonephropathy after chemotherapy for lung cancer}

A 60 year old woman with small cell cancer of the right main bronchus had the clinical, biochemical, and histological features (on biopsy) of early membranous nephropathy. Immunofluorescence studies showed granular deposits of IgG above the glomerular basement membrane. After chemotherapy with cyclophosphamide, doxorubicin, and vincristine repeat bronchoscopy and biopsy showed no tumour and the features of the membranous glomerular nephropathy resolved except for minor structural changes on light microscopy. Nineteen months after presentation she remains well, and we believe that this is the first report of complete clinical remission after cytotoxic chemotherapy.-D o'DONNELL, C P KELLY, et al, St James's Hospital, Dublin. (Accepted 12 December 1983)

\section{Reducing cytotoxic drug wastage}

The Christie Hospital has recently introduced a pharmacy based cytotoxic drug reconstitution and supply service which, at present, operates for all outpatients. Detailed surveys carried out before and after the introduction of the service have shown that the annual savings in drug wastage are now over $£ 16000$. Before the introduction of a centralised reconstituting service the wastage was above $£ 64000$ per annum for the entire hospital; this is $16 \%$ of the cytotoxic drug budget. The wastage incurred in preparing cytotoxic drugs for outpatients has now been reduced to $0 \cdot 8 \%$. Total savings at the Christie Hospital in excess of $£ 60000$ per year are possible. Even allowing for the additional two technicians required to run the service there is a substantial gain to be made.-STEPHEN BENNETT, Christie Hospital, Manchester M20 9BX. (Accepted 28 December 1983)

\section{Intraperitoneal azlocillin and aminoglycoside for dialysis associated pseudomonas peritonitis}

Peritonitis caused by pseudomonas species is relatively common in both intermittent peritoneal dialysis and continuous ambulatory peritoneal dialysis. Strains resistant to the aminoglycoside antibiotics are not uncommon and prolonged courses may be associated with ototoxicity. We have recently treated three patients with pseudomonas peritonitis with a combination of intraperitoneal azlocillin and an aminoglycoside. Clinical improvement was dramatic and dialysate cultures became negative within 48 hours of treatment with $10 \mathrm{mg}$ aminoglycoside and $250 \mathrm{mg}$ azlocillin per litre of dialysate. Treatment was well tolerated with no side effects. We suggest that this combination is more efficient and safe than an aminoglycoside alone.-A C T LEUNG, I S HENDERSON, et al, Glasgow Royal Infirmary, Glasgow G4 0SF. (Accepted 28 December 1983)

\section{Sterilisation of Esmarch elastic bandages}

To investigate methods of sterilising Esmarch bandages ten pairs were prepared by placing a strip of autoclave tape at metre intervals along their length. One of each was then wound into the customary tight roll and the other left loosely unravelled, both being autoclaved for a standard cycle. The tape on the tight roll showed only minimal steam penetration, while that packed loosely had been adequately sterilised. Nothing has been written on this topic but obviously the findings are relevant to orthopaedic surgery, where a "sterile" bandage is often used to exsanguinate a limb either before or after surgery.- - JONATHAN D HARRISON, Scarborough Hospital, Yorkshire. (Accepted 3 fanuary 1984)

"Unreviewed Reports" aims at publishing very brief findings quickly, without the usual external peer review. Each item should be no more than 100 words long, with a title of up to 10 words, only one reference, and no more than two named authors (et al is allowed). Authors of papers about side effects must have reported them to the Committee on Safety of Medicines and the manufacturers. Correspondence asking for further details about these items should be sent directly to the authors, who should be willing to supply answers. 